\title{
A method to increase the tip velocity of a shaped charge jet using a hollow cavity
}

\author{
D. R. Scheffler \& W. P. Walters \\ U.S. Army Research Laboratory, \\ Weapons and Materials Research Directorate, USA
}

\begin{abstract}
A technique is presented to increase the tip velocity of a conventional shaped charge (SC) device by employing an air cavity wave shaper which overlaps the liner. Thus, the device is a combination of an SC and an air cavity charge. Two-dimensional, axisymmetric CTH simulations were used to design the warhead. CTH is a family of computer programs developed at Sandia National Laboratories for modeling solid dynamics problems involving shock-wave propagation, multiple materials, and large deformations in one, two, or three dimensions. The baseline case was a standard 7.5-cm-diameter liner. The liner is basically a $42^{\circ}$ copper, conical liner with a tip velocity of $8 \mathrm{~km} / \mathrm{s}$. The air cavity, which overlaps the liner, increases the tip velocity of the coherent portion of the liner to about $10 \mathrm{~km} / \mathrm{s}$, with hypervelocity jet particles traveling in front of the tip with a velocity of about $14 \mathrm{~km} / \mathrm{s}$.

Keywords: shaped charge jet, wave shaper, numerical simulation, hydrocode, explosive, cavity.
\end{abstract}

\section{Introduction}

Currently used shaped charge (SC) designs (i.e., in weapon systems, oil-well completion, or drilling operations) may be designed to provide a deep hole in a target material and maximize crater volume. Increasing the tip velocity of the jet increases the depth of penetration into many targets, including most metals and geological materials. The depth of penetration is critical for most military targets and for releasing the flow of gas or oil in the oil-well completion problem. SC performance, i.e., depth of penetration, may also be improved by using a wave shaper. A wave shaper is a device that can contour or shape the detonation 
wave, causing it to impact the liner at a more favorable angle, namely nearly normal or perpendicular to the liner. Wave shapers can thus be used to alter the impact angle of the detonation wave to improve performance of the SC. They can also be used to decrease the amount of explosive required to form a fast jet. Wave shapers have been made of many materials, including metals, plastics, concrete, and air. Usually, the intent is to contour the detonation wave to move around the wave shaper to impact the liner at a favorable angle. Here, a hollow wave-shaper concept is examined where the hollow volume overlaps the apex of the SC liner. The goal is to produce low-mass, high-velocity particles to simulate micrometeorite particles which may impact spacecrafts. A series of five hydrocode simulations are presented, where one simulation is a baseline SC without a hollow cavity to which all other simulations are compared. The SC concept that uses a hollow cavity overlapping the apex of the SC liner is further detailed in Walters and Scheffler [1].

The simulations were performed using the CTH hydrocode [2], a state-of-theart, second-order accurate, Eulerian hydrocode developed by Sandia National Laboratories. This code, which is capable of solving complex problems in shock physics in one, two, or three dimensions, provides several constitutive models, including an elastic-perfectly plastic model with provisions for work hardening and thermal softening, the Johnson-Cook model [3], the Zerrilli-Armstrong model [4], the Steinberg-Guinan-Lund model [5,6], an undocumented powerlaw model, and others. High-explosive detonation can be modeled using the programmed burn model, the Chapman-Jouguet volume burn models, or the history variable reactive burn model [7]. Several equation-of-state (EOS) options are available, including tabular (i.e., SESAME), analytical (ANEOS), MieGrüneisen, and Jones-Wilkins-Lee (JWL) [8]. Material failure occurs when a threshold value of tensile stress or hydrostatic pressure is exceeded. In addition, the Johnson-Cook failure model [9] is also available. When failure occurs in a cell, void is introduced until the stress state of the cell is reduced to zero. Recompression is permitted. To reduce the diffusion typically encountered in Eulerian simulations, several advanced material interface tracking algorithms are provided, including the high-resolution interface tracking algorithm (available for two-dimensional [2-D] simulations only), the simple line interface calculation algorithm [10], and the Sandia-modified Youngs' reconstruction algorithm [11].

\section{Simulation setup}

A series of five 2-D simulations using the March 1999 version of the CTH hydrocode were performed using cylindrical symmetry for each of the SC geometries shown in Figures 1-5. Figure 1 shows the baseline SC. The baseline $\mathrm{SC}$ consists of a copper liner that has an apex angle of $42^{\circ}$, a liner thickness of $0.225 \mathrm{~cm}$, and a base diameter of $7.5 \mathrm{~cm}$. The SC liner had a mass of $232 \mathrm{~g}$. The unconfined explosive was 78/22 Octol; the JWL EOS was used. Explosive detonation was accomplished using a simple programmed burn model and point initiated at the rear of the SC. 
All simulations employed axisymmetry and used the same computational mesh. The mesh consisted of $207 \times 2163$ cells, with the origin of the coordinate system at the base of the SC liner. A constant grid of purely square cells, with a cell size of $0.0241 \times 0.0241 \mathrm{~cm}$, was used. Simulations were carried out until the lead jet particles left the computational mesh.

\section{Simulation results}

Figure 6 shows the shaped charge jet (SCJ) for the baseline case. At $60 \mu \mathrm{s}$, the SCJ is still coherent and has a typical shape for a jet before it particulates. The left side of Figure 6 is a pressure plot showing only compression, and the right side is a material plot showing only the jet material (the explosive material has been discarded from the simulation at an earlier time). The axial velocity profile is shown in Figure 7. Figure 7 shows the tip of the SCJ travelling at approximately $8 \mathrm{~km} / \mathrm{s}$. Also examined was the maximum axial pressure experienced by the SCJ, as seen in Figure 8 . Figure 8 shows the maximum pressure of $85 \mathrm{GPa}$ at $20 \mu \mathrm{s}$. (It should be noted the axial pressure plots were examined at $1-\mu$ s intervals and that the actual peak pressure may have been missed.) The SCJ tip velocity and maximum pressure are used for comparing the remaining examined charges. It was postulated that the high pressure in the air cavity resulted in the higher jet tip velocity.

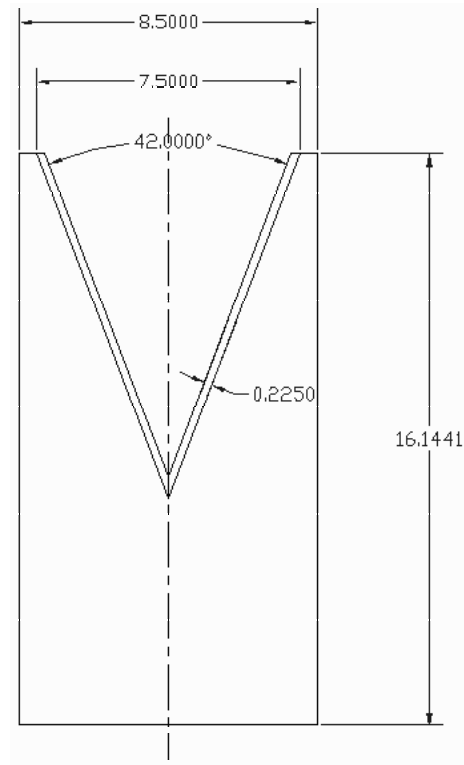

All dimensions in $\mathrm{cm}$

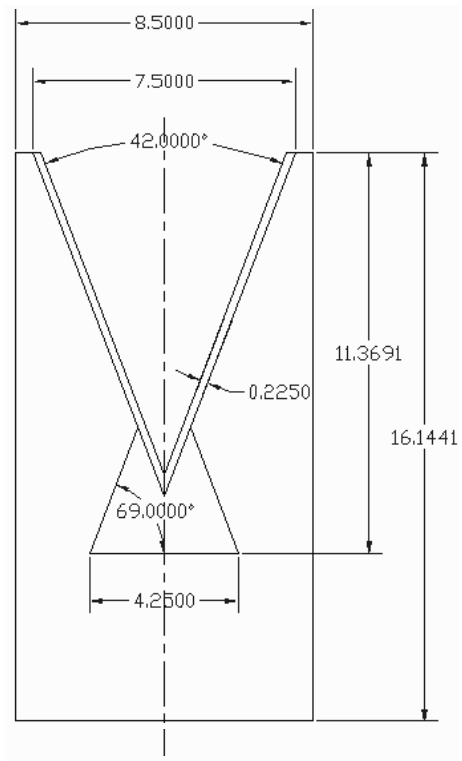

All dimensions in $\mathrm{cm}$

Figure 1: Baseline SC configuration. Figure 2: Case 1: SC with baseline cavity overlapping liner. 


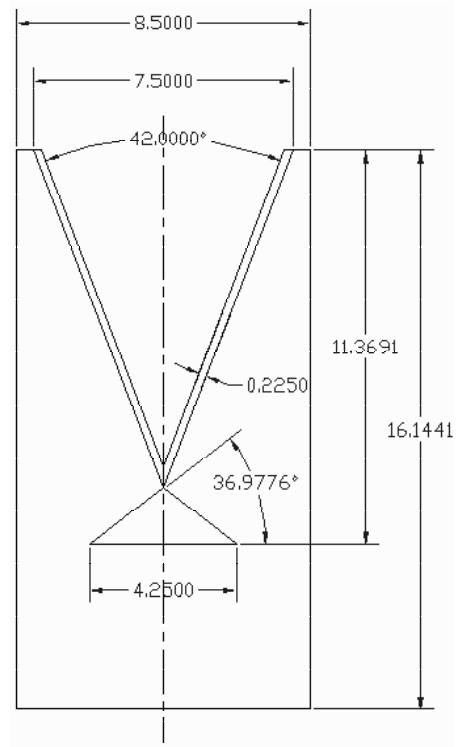

All dimensions in $\mathrm{cm}$

Figure 3: Case 2: SC with cavity wave shaper, no overlap.

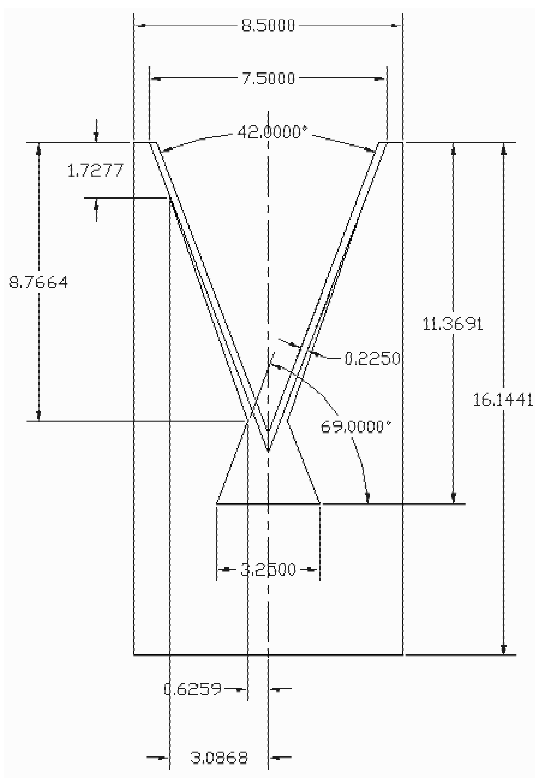

All dimensions in $\mathrm{cm}$

Figure 4: Case 3: SC with reduced and modified cavity overlapping liner. 


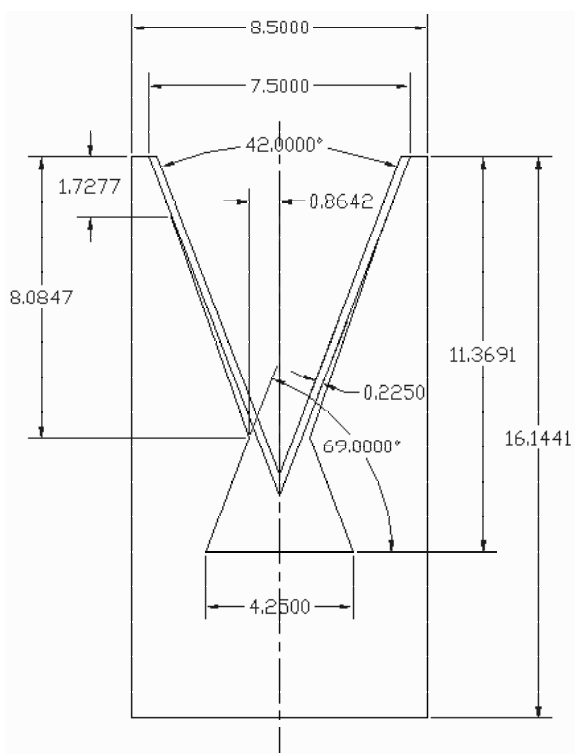

All dimensions in $\mathrm{cm}$

Figure 5: Case 4: SC with modified baseline cavity overlapping liner.

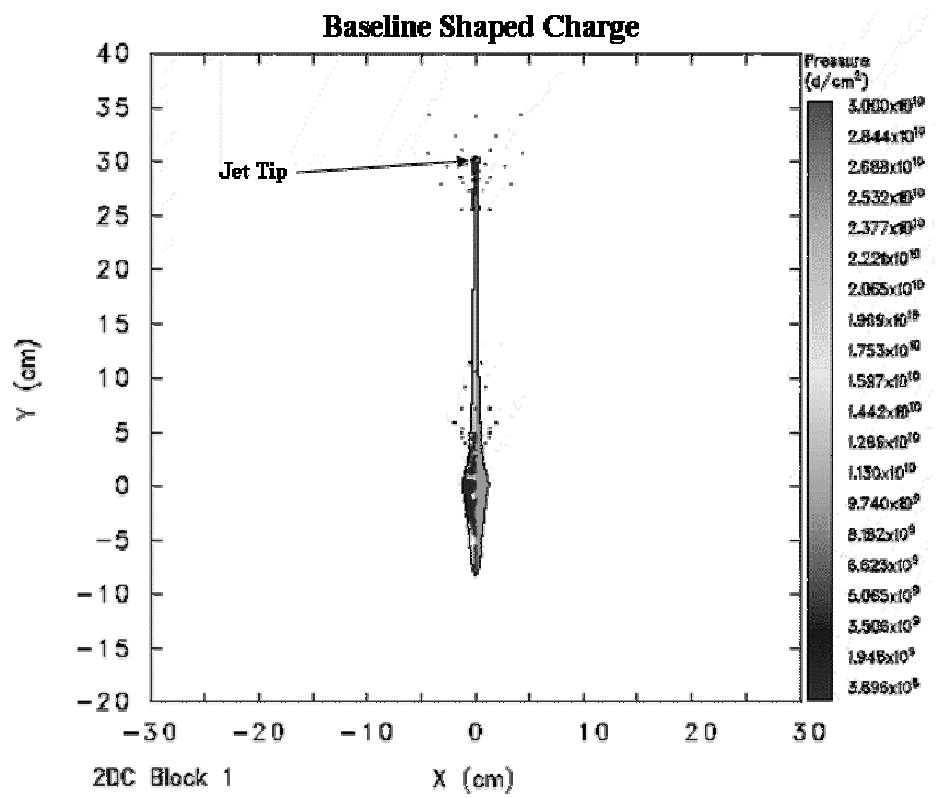

Figure 6: Baseline $\mathrm{SC}$ at $60 \mu \mathrm{s}$. 


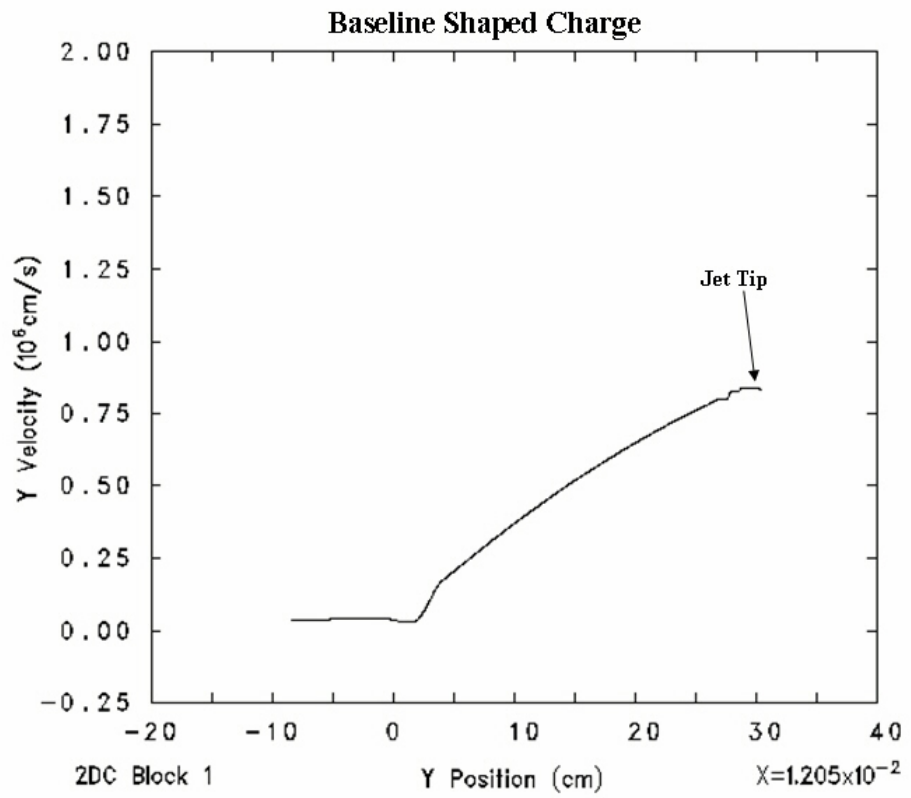

Figure 7: Axial velocity for baseline SC at $60 \mu \mathrm{s}$.

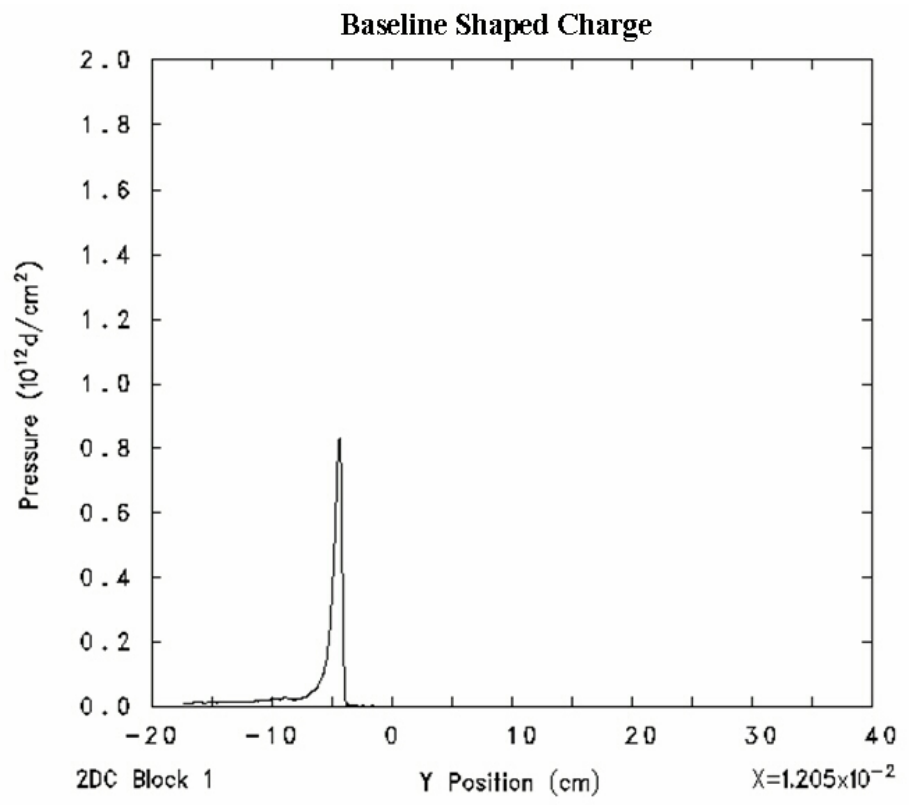

Figure 8: Axial pressure for baseline SC at $20 \mu \mathrm{s}$. 
Figure 9 shows the geometry and pressure plot for the Case $1 \mathrm{SCJ}$ at $40 \mu \mathrm{s}$, as shown in Figure 2. Case 1 shows the wave shaper cavity overlapping the SC liner. Unlike the baseline SC, Figure 9 shows a coherent SCJ, with hypervelocity particles ahead of the main jet tip. The axial velocity profile of the SCJ, shown in Figure 10, shows that the tip velocity is approximately $10 \mathrm{~km} / \mathrm{s}$, with the leading hypervelocity particles travelling much faster. The maximum velocity of the lead particle is approximately $14 \mathrm{~km} / \mathrm{s}$. Figure 11 shows the axial pressure profile along the jet at $12 \mu \mathrm{s}$, the time when maximum pressure was observed. The maximum pressure was approximately $185 \mathrm{GPa}$. All other SC cases with wave shapers, whether the hollow cavity overlaps the apex of the SCJ liner or not, had similar jet profiles to that shown in Figure 9. Due to page limitations, the results of all cases are simply summarized in Table 1.

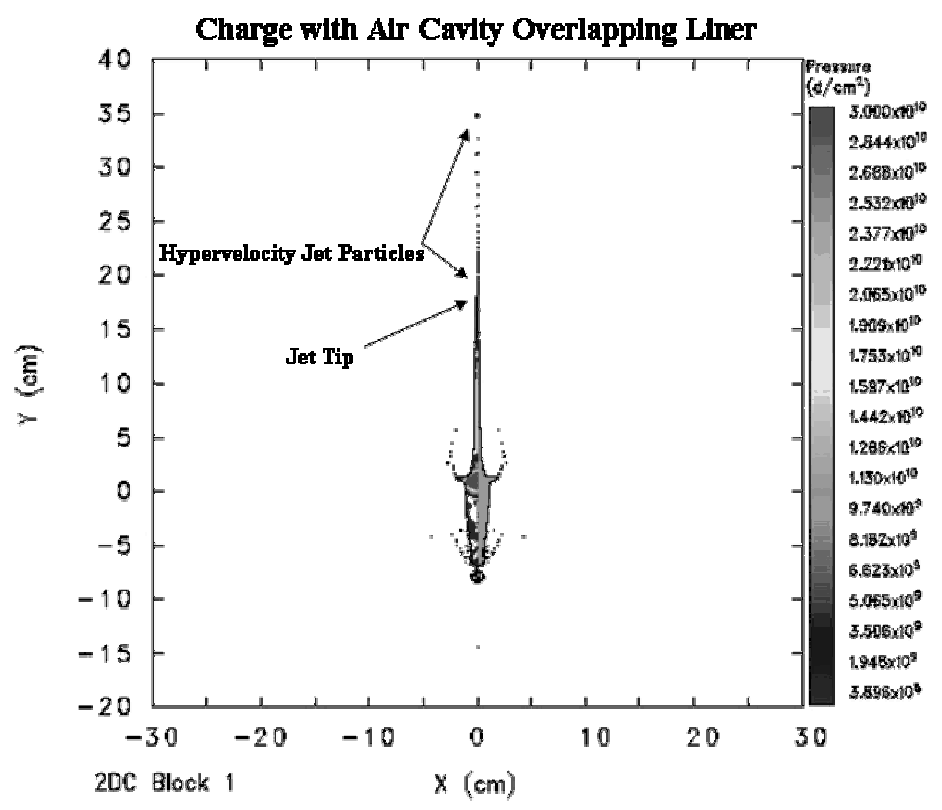

Figure 9: $\quad$ Case $1 \mathrm{SC}$ at $40 \mu \mathrm{s}$.

The Case 2 SC, Figure 3, does not have a cavity that overlaps the apex of the copper liner. Rather, the conical cavity's apex meets at the liner's apex. From Table 1, the Case 2 SCJ gives a slightly higher SCJ tip velocity than the baseline case, namely $8.7 \mathrm{~km} / \mathrm{s}$. Though the cavity of Case 2 did not overlap the copper liner, it still produced hypervelocity particles similar to those shown for Case 1 in Figure 9. The approximate maximum particle velocity was $12 \mathrm{~km} / \mathrm{s}$. The maximum pressure seen for the Case $2 \mathrm{SCJ}$ along its axis occurred at $9 \mu \mathrm{s}$ and was $135 \mathrm{GPa}$. While this charge represented an improvement in performance over the baseline SC, it used more explosive than Case 1 and did not match the Case $1 \mathrm{SC}$ performance. 
106 Computational Ballistics III

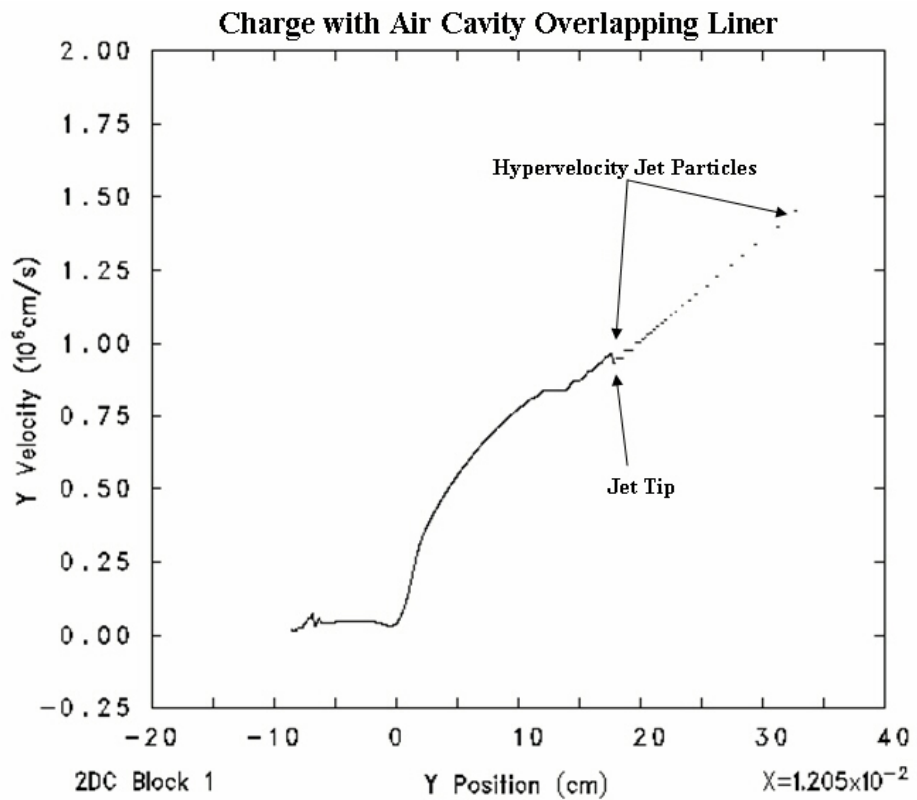

Figure 10: Axial velocity for Case $1 \mathrm{SC}$ at $40 \mu \mathrm{s}$.

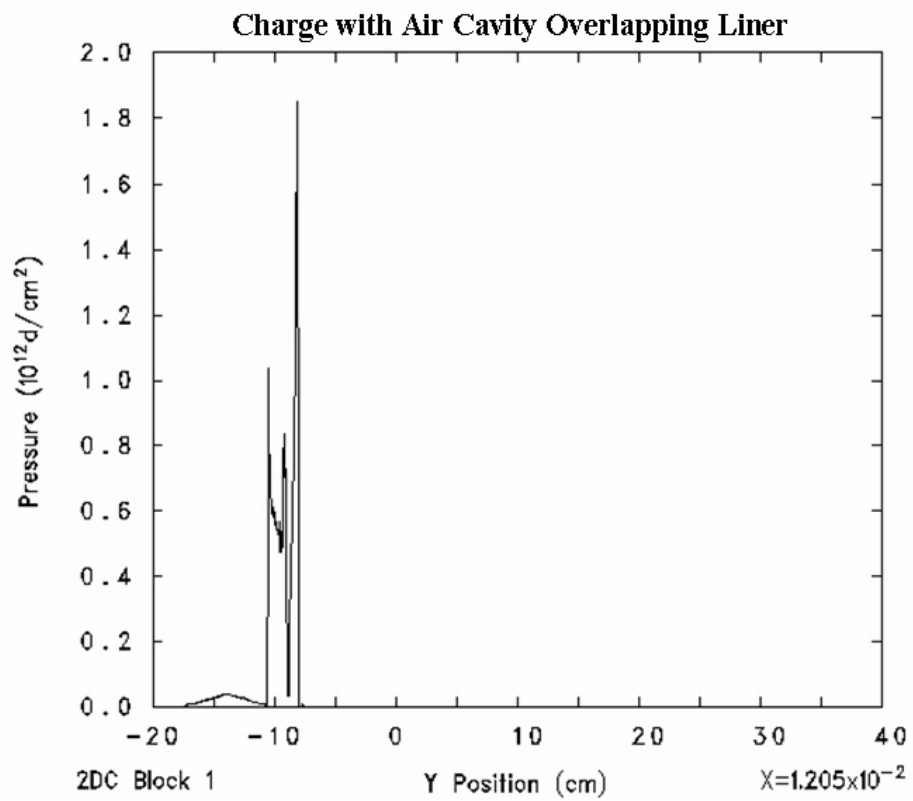

Figure 11: Axial pressure for Case $1 \mathrm{SC}$ at $12 \mu \mathrm{s}$. 
Table 1: $\quad$ Comparison of simulation results.

\begin{tabular}{|c|c|c|c|c|}
\hline Case \# & $\begin{array}{c}\text { Charge } \\
\text { Mass } \\
(\mathbf{g})\end{array}$ & $\begin{array}{c}\text { Max. Particle } \\
\text { Velocity } \\
(\mathbf{k m} / \mathbf{s})\end{array}$ & $\begin{array}{c}\text { Jet Tip } \\
\text { Velocity } \\
(\mathbf{k m} / \mathbf{s})\end{array}$ & $\begin{array}{c}\text { Max. Pressure } \\
\text { \& Time at Max. } \\
(\mathbf{G P A} \text { @ } \boldsymbol{\mu s})\end{array}$ \\
\hline Baseline & 1406 & N/A & 8.0 & $85 @ 20$ \\
\hline 1 & 1363 & 14.0 & 10.0 & $185 @ 12$ \\
\hline 2 & 1392 & 12.0 & 8.7 & $135 @ 9$ \\
\hline 3 & 1373 & 11.2 & 8.7 & $165 @ 10$ \\
\hline 4 & 1340 & 12.5 & 8.9 & $115 @ 11$ \\
\hline
\end{tabular}

$\mathrm{N} / \mathrm{A}=$ not applicable.

For the Case $3 \mathrm{SC}$, the cavity again overlaps the apex of the liner and has additional explosive removed along the liner, as shown in Figure 4. This represents a reduced and modified cavity from Case 1 as shown in Figure 2, and thus has more explosive mass than Case 1 (see Table 1). From Table 1, it can be seen that while the velocity-based performance, maximum hypervelocity particle velocity, and pressure does not match the performance of Case 1, it nevertheless represents an improvement over the baseline SC. The jet tip velocity was the same as Case 2, which did not overlap the liner apex; however, the maximum hypervelocity particle velocity was less, about $11.2 \mathrm{~km} / \mathrm{s}$ compared to $12 \mathrm{~km} / \mathrm{s}$.

For Case 4, Case 1 (Figure 2) was modified by removing explosive material along the liner, as shown in Figure 5. Of all the SCs examined, Case 4 used the least amount of explosive mass, about $1340 \mathrm{~g}$, as listed in Table 1 . The Case 4 $\mathrm{SC}$ was the second best in performance when measured in terms of maximum particle velocity, jet tip velocity, and maximum pressure. It was exceeded only by Case 1 . However, probably none of the cases studied represented the optimal air cavity shape.

\section{Conclusions}

The results of five axisymmetric simulations were presented. The first simulation represented a standard SC without a hollow wave shaper. The second simulation, Case 1, represented the baseline hollow wave shaper cavity which overlaps the apex of the SC liner. While wave shapers, including hollow cavities, have been in use for some time, it was shown that by using a hollow cavity overlapping the apex of the liner, improvement in SCJ performance, as measured in terms of jet tip velocity, could be obtained. The generation of higher velocity particles preceding the jet tip could also be obtained. The expansion of the overlapping cavity by removing explosive along the side of the liner showed no real advantage.

\section{References}

[1] Walters, W.P. \& Scheffler, D.R., Shaped charge explosive device and method of making the same. Patent 6,983,698, 2006. 
[2] McGlaun, J.M., Thompson, S.L. \& Elrick, M.G., CTH: a threedimensional shock wave physics code. International Journal of Impact Engineering, 10(1-4), pp. 351-360, 1990.

[3] Johnson, G.R. \& Cook, W.H., A constitutive model and data for metals subjected to large strains, high strain rates and high temperatures. Proc. of the $7^{\text {th }}$ Int. Symp. on Ballistics, The Hague, The Netherlands, pp. 541-548, 1983.

[4] Zerilli, F.J. \& Armstrong, R.W. Dislocation-mechanics-based constitutive relations for material dynamics calculations. Journal of Applied Physics, 61(5), pp. 1816-1825, 1987.

[5] Steinberg, D.J., Cochran, S.G. \& Guinan, M.W., A constitutive model for metals applicable at high-strain rate. Journal of Applied Physics, 51(3), pp. 1498-1504, 1980.

[6] Steinberg, D.J. \& Lund, C.M., A constitutive model for strain rates from $10^{-4}$ to $10^{6} \mathrm{~s}^{-1}$. Journal of Applied Physics, 65(4), pp. 1528-1533, 1989.

[7] Kerley, G.I., CTH equation of state package: porosity and reactive burn models. SAND92-0553, Sandia National Laboratories, Albuquerque, NM, 1992.

[8] Lee, E.L., Hornig, H.C. \& Kury, J.W., Adiabatic expansion of high explosive detonation products. UCRL-50422, Lawrence Livermore National Laboratory, Livermore, CA, 1968.

[9] Johnson, G.R. \& Cook, W.H., Fracture characteristics of three metals subjected to various strains, strain rates, temperatures, and pressures. Journal of Engineering Fracture Mechanics, 21(1), pp. 31-48, 1985.

[10] Noh, W.F. \& Woodward, P., SLIC (simple line interface calculation). Lecture Notes in Physics, 59, Springer-Verlag, 1976.

[11] Bell, R.L. \& Hertel Jr., E.S., An improved material interface reconstruction algorithm for eulerian codes. SAND92-1716, Sandia National Laboratories, Albuquerque, NM, 1992. 